\title{
The Effect of Knowledge Management on Empowerment of Youth and Sports General Directorate of Mazandaranprovince
}

\author{
Fataneh Asadollahzadeh* \\ Department of Physical Education, Islamic Azad University, Iran \\ *Corresponding author: Fataneh Asadollahzadeh, Master of bachelor, Department of Physical Education, Qaemshahr branch, Islamic Azad University, \\ Qaemshahr, Iran
}

Submission: 監 May 22, 2018; Published: 悳 June 11, 2018

\begin{abstract}
The purpose of this study was to investigate the effect of knowledge management on the empowerment of employees of the Ministry of Sport and Youth in Mazandaran province. The research method was field survey and has been done on field based. The statistical population of this study is all employees of the Ministry of Sports and Youth of Mazandaran province, which were selected using the Morgan table in 129 simple random samples. The knowledge empowerment and management questionnaire was used to collect information. Regarding the analysis and analysis of the multivariate regression test, the SPSS23 software was used. The findings of the research showed that there is a positive and significant relationship between the components of knowledge management and empowerment and knowledge management has an impact on empowerment of employees.
\end{abstract}

Keywords: Knowledge management; Empowerment; Staff; Department of sports and youth; Mazandaran

\section{Introduction}

In today's world, the success and prosperity of various organizations and societies depends on the application and optimal use of management science and its capabilities; as a result, organizations and sports managers cannot stand apart from this [1]. Given the fact that organizations are witnessing rapid and unpredictable changes in the environment, in such situations, managers do not have much opportunity to control the staff and should devote their most time and effort to identifying the internal and external environment and other tasks. Staffs are left to do their everyday task. Hence, the most important source of competitive advantage in organizations is committed, excited and responsible employees, therefore, empowerment is considered as the most important challenge of managers in the present era [2]. The organization of physical education is the main institution that influences all sports activities of the country, which plays a significant role in promoting the level of sport and health, sustainable development, prominence of the country, etc., and like other organizations in order to achieve their organizational goals, it requires continuous learning in all Levels of work of the organization [3].

Since physical education plays a pivotal role in improving and improving the physical and mental health of the various strata of society, especially the younger generation, as well as due to the particular sport situation (especially the championship and professional sport of the country), which is guided by the hand of general office of sport and the youth, It is vital for the whole organization to have an effective and effective role to play, and this cannot be possible, except that the organization employs efficient managers and staff, and to utilize the capabilities of managers and For employees, this is a critical issue; to provide a desirable environment for human resource activity, resulting in their satisfaction and to be developed and stimulated to use all their power and thought in order to succeed in the organization and achieve the goals of the organization [4]. Skilled and efficient human resources are one of the most important tools for reaching the goals of the organization, because human resources play an important role in increasing and decreasing the productivity of the organization [5]. Considering that efficient human resources are the main indicator of the superiority of an organization to other organizations, the emphasis on effective and efficient human resources is also increasing [6].

Gradually, organizations need something beyond information management for the actual use of employee skills [7]. Knowledge Management is a range of activities that are used to manage 
exchange, create, or promote intellectual capital at macro level [8]. The goal of KM is to create a common understanding through the alignment of people, processes and tools within the structure and organizational culture to enhance collaboration and interaction between managers and subordinates [9]. Other concepts raised in the context of human resource development are empowerment [10]. In order to empower the staff of an organization, it is necessary to develop ways to explore the competency of individuals in order to develop capabilities and maximize the opportunity for qualified individuals to influence all aspects of the organization's work, including the distribution of competences focused on the use of principles and strategies. The organization is not effective in empowerment that focuses on the distribution of competences based on competence and can enhance knowledge management [8]. Empowerment is closely linked with continuous improvement, competence development, change in behavior and improvement in organizational elements, and can be improved by improving the performance of staff and fixes the survival of organization $[11,12]$.

There are various ambiguities about the effects of empowerment on knowledgemanagement; in recentyears, knowledgemanagement has become a critical issue in the literature of both industrial and service [13]. Knowledge management in today's advanced societies is considered as one of the tools for advancement, which can lead to the growth of impotent talent and high efficiency in manpower, and enable managers with entrepreneurship and powerful staff to lead knowledge management [14]. On the other hand, it is argued that today the main concern of the organization is the use of human resources as the most important sources of the organization for organizational purposes. In today's competitive world, if organizations cannot make a committed and conscientious body for their own organization, it will definitely not be a fate to defeat against others. Human resources, as the most important sources of the organization and at the same time as the most sensitive source, have always been the main discussion of the scientific schools of management [15].

On the other hand, knowledge managementis a range of activities that are used to manage exchange, create, or promote intellectual capital at macro level [7]. Given that knowledge management is strategies and processes that are capable of generating and streamlining knowledge in order to create the expectations of the organization, customers and users throughout the organization, it is therefore considered to be of great importance [16]. In other words, knowledge management makes value by converting human capital into organized intellectual property for the organization [17]. Therefore, empowerment, based on qualification enhancement and on condition of having the opportunity, is able to implements the goals of the staff and the organization and controls its results. It also leads to optimal performance and leads to self-reliance and selfrewarding self-esteem and increasing self-esteem in which case, intellectual freedom and physical movement are produced and a sense of curiosity, research, learning and the action is provoked [18].

Therefore, there are various ambiguities about the effects of empowerment on knowledge management in organizations, government departments, and in particular physical education departments. Along with the progression of science, empowerment, especially in sports and youth, plays an important role in this regard, whereas, one can see the increase of scientific ability in different areas of the organization and as a result we have the development of science and the progress of society, and as a result of empowerment of employees, we will see knowledge management. Therefore, despite the many studies that have been done about the effects of knowledge management on empowerment, their role is still on the curriculum, and it is not clear how much knowledge management is effective in empowering employees. Therefore, considering the effective role of knowledge management in increasing the efficiency and effectiveness of organization activities, this study was conducted to answer the following question: How is knowledge management and staffing capabilities of the General Office of Sports and Youth of the province? How does knowledge affect the empowerment of staff of the General Office of Sports and Youth of Mazandaran Province?

\section{Methodology}

The research method was descriptive-survey and correlation type was done as field experiment. The statistical population of this research included all staff of the Sports and Youth Department of Mazandaran province (154 people, 69 women and 84 men). According to Morgan's table for homogeneity of the community and the research sample, the sample size of the study was 59 women and 70 men ( $n=129)$. To collect data, Kamkari \& Karimnezhad [19] Empowerment Questionnaire $(\alpha=0.75)$, Kamkari \& Shokrzadeh [20] Knowledge Management Questionnaire $(\alpha=0.94)$ were used. The multivariate regression model using The SPSS $_{23}$ software and the prediction of knowledge management through empowerment are discussed.

The formula is as follows:

$\mathrm{b} 1 \mathrm{x} 1+\mathrm{b} 2 \times 2+\mathrm{b} 3 \times 3+b 4 \times 4+b 5 \times 5+a=y$

(Knowledge application $\mathrm{x} 1)+($ Knowledge distribution $\mathrm{x} 2)+($ Knowledge presentation $\mathrm{x} 3)+($ Knowledge verification $\mathrm{x} 4)+($ Knowledge creation $\mathrm{x} 5)+$ Fixed number=Empowerment

\section{Findings}

The results of the study showed that $54.26 \%$ of men, $76.77 \%$, of the age group had the highest number with $36.66 \%$ in the age range of 25-30 years and the lowest number was $6.98 \%$ in the age range of more than 50 years. $41.31 \%$ have a bachelor's degree. $72.99 \%$ have studied in the field of physical education. Also, 55.18\% have a service record of 5 to 10 years (Table 1). 
According to the above table, with emphasis on the amount of F obtained, it can be argued that there is a significant relationship between all dimensions of knowledge management with empowerment at the level of $\alpha=0.05$. In other words, there is the ability to predict "knowledge management and the dimensions of that knowledge" through "empowerment" (Table 2).

Table 1: Multivariate regression to predict "knowledge management components" through "empowerment".

\begin{tabular}{|c|c|c|c|c|c|c|}
\hline Variable & Source of Change & Sum of Squares & DF & Averages of Squares & $\mathbf{F}$ & Sig. \\
\hline \multirow{2}{*}{ Create knowledge } & Regression & 404.34 & 1 & 404.34 & 4.26 & .039 \\
\hline & Remaining & 9016.02 & 128 & 94.90 & & \\
\hline \multirow{2}{*}{$\begin{array}{l}\text { Knowledge } \\
\text { verification }\end{array}$} & Regression & 1089.55 & 1 & 272.38 & 2.68 & .026 \\
\hline & Remaining & 9236.22 & 28 & 98.25 & & \\
\hline \multirow{2}{*}{$\begin{array}{l}\text { Providing } \\
\text { knowledge }\end{array}$} & Regression & 1512.31 & 1 & 378.07 & 3.41 & .004 \\
\hline & Remaining & 9218.18 & 28 & 99.12 & & \\
\hline \multirow{2}{*}{$\begin{array}{l}\text { Knowledge } \\
\text { Distribution }\end{array}$} & Regression & 1492.61 & 1 & 373.15 & 4.070 .039 & .002 \\
\hline & Remaining & 8437.16 & 28 & 89.75 & & \\
\hline \multirow{2}{*}{$\begin{array}{l}\text { Knowledge } \\
\text { application }\end{array}$} & Regression & 1970.03 & 1 & 492.5 & 3.240 .026 & .005 \\
\hline & Remaining & 11245.5 & 28 & 124.95 & & \\
\hline
\end{tabular}

Table 2: Regression coefficients associated with knowledge creation component.

\begin{tabular}{|c|c|c|c|c|c|}
\hline Criterion Variable & Predictive Variables & Amount Beta & Beta Coefficient & t & Sig. \\
\hline \multirow{4}{*}{ Knowledge Creation } & Meaningful & 0.79 & 0.2 & 1.06 & 0.039 \\
\cline { 2 - 6 } & Competence & 0.45 & 0.2 & 0.31 & 0.064 \\
\cline { 2 - 6 } & Autonomy & 0.1 & 0.03 & -0.99 & 0.723 \\
\cline { 2 - 6 } & Influence & 0.34 & -0.12 & 0.225 \\
\hline
\end{tabular}

Considering the multi-variable regression coefficients with simultaneous login method and obtained regression coefficients, it can be argued that there is a significant positive relationship between the components of "meaningful" and "competence" with "knowledge validation". By increasing the components of "meaningful" and "competence", "Knowledge Creation" also increases and with their reduction, "Knowledge Creation" also decreases (Table 3).

Table 3: Regression coefficients associated with Knowledge verification component.

\begin{tabular}{|c|c|c|c|c|c|}
\hline Criterion Variable & Predictive Variables & Amount Beta & Beta Coefficient & t & Sig. \\
\hline \multirow{4}{*}{ Knowledge verification } & Meaningful & 1.26 & 0.32 & 2.4 & 0.014 \\
\cline { 2 - 6 } & Competence & 1.07 & 0.33 & 0.08 & 0.03 \\
\cline { 2 - 6 } & Autonomy & 0.07 & 0.01 & 0.61 & 0.871 \\
\cline { 2 - 6 } & Influence & 0.35 & 0.1 & 0.589 \\
\hline
\end{tabular}

Considering the multi-variable regression coefficients with simultaneous login method and obtained regression coefficients, it can be argued that there is a significant positive relationship between the components of "meaningful" and "competence"

with "Knowledge verification". By increasing the components of "meaningful" and "competence", "Knowledge verification" also increases and with their reduction, "Knowledge verification" also decreases (Table 4).

Table 4: Regression coefficients associated with providing knowledge component.

\begin{tabular}{|c|c|c|c|c|c|}
\hline Criterion Variable & Predictive Variables & Amount Beta & Beta Coefficient & t & Sig. \\
\hline \multirow{4}{*}{ Providing knowledge } & Meaningful & 1.27 & 0.32 & 1.5 & 0.011 \\
\cline { 2 - 6 } & Competence & 0.99 & 0.3 & 0.87 & 0.064 \\
\cline { 2 - 6 } & Autonomy & 0.44 & 0.1 & 0.8 & 0.283 \\
\cline { 2 - 6 } & Influence & 0.45 & 0.13 & 0.325 \\
\hline
\end{tabular}

Considering the multi-variable regression coefficients with simultaneous login method and obtained regression coefficients, it can be argued that there is a significant positive relationship between the components of "meaningful" and "competence"

with "Providing knowledge". By increasing the components of "meaningful" and "competence", "Providing knowledge" also increases and with their reduction, "Providing knowledge" also decreases (Table 5). 
Table 5: Regression coefficients associated with knowledge distribution component.

\begin{tabular}{|c|c|c|c|c|c|}
\hline Criterion Variable & Predictive Variables & Amount Beta & Beta Coefficient & t & Sig. \\
\hline \multirow{4}{*}{$\begin{array}{c}\text { Knowledge } \\
\text { Distribution }\end{array}$} & Meaningful & 1.2 & 0.32 & 2.6 & 0.008 \\
\cline { 2 - 6 } & Competence & 1.09 & 0.35 & 1.36 & 0.019 \\
\cline { 2 - 6 } & Autonomy & 0.59 & 0.15 & 0.69 & 0.135 \\
\cline { 2 - 6 } & Influence & 0.37 & 0.11 & 0.388 \\
\hline
\end{tabular}

Considering the multi-variable regression coefficients with simultaneous login method and obtained regression coefficients, it can be argued that there is a significant positive relationship between the components of "meaningful" and "competence" with "Knowledge Distribution". By increasing the components of "meaningful" and "competence", "Knowledge Distribution" also increases and with their reduction, "Knowledge Distribution" also decreases (Table 6).

Table 6: Regression coefficients associated with knowledge application component.

\begin{tabular}{|c|c|c|c|c|c|}
\hline Criterion Variable & Predictive Variables & Amount Beta & Beta Coefficient & t & Sig. \\
\hline \multirow{4}{*}{$\begin{array}{c}\text { Knowledge } \\
\text { Distribution }\end{array}$} & Meaningful & 1.37 & 0.3 & 2.21 & 0.019 \\
\cline { 2 - 6 } & Competence & 1.12 & 0.31 & 0.84 & 0.058 \\
\cline { 2 - 6 } & Autonomy & 0.5 & 0.1 & 0.94 & 0.285 \\
\cline { 2 - 6 } & Influence & 0.69 & 0.18 & 0.376 \\
\hline
\end{tabular}

Considering the multi-variable regression coefficients with simultaneous login method and obtained regression coefficients, it can be argued that there is a significant positive relationship between the components of "meaningful" and "competence" with "Knowledge application". By increasing the components of "meaningful" and "competence", "Knowledge application" also increases and with their reduction, "Knowledge application" also decreases.

The prediction of knowledge management through empowerment is discussed. The formula is as follows:

$$
(0.039 X 1)+(0.026 \quad \mathrm{X} 2)+(0.004 \quad \mathrm{X} 3)+(0.002 \quad \mathrm{X} 4)+(0.005
$$

$\mathrm{X} 5)+\mathrm{a}=$ Empowerment

\section{Discussion and Conclusion}

Considering the results obtained from the first hypothesis, it can be argued that there is a significant positive relationship between the "meaningful" component and the "creation of knowledge". By increasing the "meaningful" component, "knowledge creation" also increases, and with its decrease, the "creation of knowledge" also decreases. The results of this study are consistent with the results of Farahani \& Falahati [20], Desheeri (2013), Rastadmehr et al. [8], Esfandiari \& Adabi [5], Hasani \& Sheikh Esmaeili [21], Shaki et al. [22]. On the other hand, Farahani \& Falahati [20] suggested that meaningful component has a positive and significant relationship with organizational entrepreneurship that has close relationship with knowledge management in organization. Sahraee (2013) and Rastadmehr et al. [8], Esfandiari \& Adabi [5], Hasani \& Sheikh [21], Schakeri et al. (2016) stated that increasing the meaningful component increases knowledge creation and decreases it, Knowledge creation also decreases. Also, Nourian [23] consider the effectiveness (meaningful) of effectiveness as effective facilitator of participatory management on the organizational empowerment of effective employees.

According to the results of the second hypothesis, there is a significant positive correlation between the components of "meaningfulness" and "competence" with "knowledge validation". By increasing the components of "meaningfulness" and "competence", "confirmation of knowledge" also increases and with their reduction, "confirmation of knowledge" also decreases. The results of this research are consistent with the descriptive results of Sahraee (2013), Rastadmehr et al. [8], Esfandiari \& Adabi [5], Hasni \& Sheikh Esmaeili [2], Shakki et al. [22]. Farahani \& Falahati [20] suggested that the competency component has a positive and significant relationship with organizational entrepreneurship that has close relationship with knowledge management in the organization. Sahraee (2013) and Rastadmehr et al. [8], Esfandiari \& Adabi [5], Hasani \& Sheikh Esmaeili [2], Schakeri et al. (2016) stated that with increasing the meaningfulness and competence component, the confirmation of knowledge also increased and with decreasing, they also decrease the knowledge validation.

According to the results of the third hypothesis, there is a significant positive relationship between the "meaningful" component and the "presentation of knowledge". Thus, with the increase of the "meaningful" component, the "presentation of knowledge" also increases and with the decrease, the "presentation of knowledge" also decreases. The results of this research are consistent with the Sahraee (2013), Rastadmehr et al. [8], Esfandiari \& Adabi [5], Hasni \& Sheikh Esmaeili [2], Shakki et al. [22]. Sahraee (2013), Rastadmehr et al. [8], Esfandiari \& Adabi [5], Hasni \& Sheikh Esmaeili [2], Shakki et al. [22] stated that by increasing the meaningful component, the knowledge supply also increased and with decreasing them, the presentation of knowledge also decreases. 
According to the results of the fourth hypothesis, there is a significant positive correlation between the components of "meaningfulness" and "competence" with the "distribution of knowledge". By increasing the components of "meaningfulness" and "competence", the "distribution of knowledge" also increases and with their decrease, the "distribution of knowledge" also decreases. The results of this study were compared with the results of Ghorbanizadeh \& Khaleghinia [24], Sahraee (2013), Rastadmehr et al. [8], Esfandiari \& Adabi [5], Hasni \& Sheikh Esmaeili [2], Shakki et al. [22] And colleagues. The researchers stated that by increasing the meaningfulness and competence component, the distribution of knowledge also increases and with their decrease, the distribution of knowledge also decreases. The results are in conflict with Keshavarz [25] research results as barriers and facilitators of sharing and knowledge distribution in the organizations. Creating knowledge affects the organization and leads to a reduction in organizational costs and, as a result, solves organizational problems and removes barriers to exploitation. Ghorbani Zadeh \& Khaleghnia [24] stated that there is a positive and significant relationship between the transfer of knowledge, on the one hand, and the ability of employees to make decisions, the acceptance of responsibility for decision-making by staff, access of staff to decision-making and implementation tools and, finally, acceptance of decision-making responsibilities On the other hand.

According to the results of the fifth hypothesis, there is a significant positive correlation between the "meaningful" component and the "application of knowledge". Thus, with the increase of the "meaningful" component, "knowledge use" also increases and with its decrease, the "use of knowledge" also decreases. The results of this research are consistent with the Sahraei (2013), results Rastadmehr et al. [8], Esfandiari \& Adabi [5], Hasni \& Sheikh Esmaeili [2], Shakki et al. [22]. They stated that by increasing the meaningful component, the use of knowledge also increased, and by reducing them, the use of knowledge also decreases. Finally, the results of this research in response to the main question of research on the effect of knowledge management dimensions on empowerment of staff of the General Department of Sports and Youth of Mazandaran province showed that all aspects of knowledge management affect the empowerment of employees of the General Department of Sports and Youth in Mazandaran province. However, in There are some differences in the dimensions of empowerment. The results of this study were compared with the results of Saedi \& Nadalipour [26], Mousavi [27], Sahraei (2013), Rastadmehret al. [8], Esfandiari \& Adabi [5], Baker [28], Kirkman \& Rosen [29] Mostafa-Pour et al. (2012), Razaghi et al. [30], Hasani \& Sheikh Esmaeili [2], Shakki et al. [22].

Saedi \& Nadalipour [26] state that knowledge management is considered as one of the new management approaches in organizational development of the new century and helps organizations to more efficiently and efficiently exploit their resources. The most important assets of an organization, physical resources, organizational resources and human resources are intelligent and knowledgeable employees who are engaged in new organizational processes, new technology and the development of new products. Innovative efforts are the result of investment in human resource management and knowledge management. Mousavi [28] also argued that the culture of sharing and distributing knowledge and understanding of human resources from knowledge, staff training and support for knowledge management projects and targeting knowledge transfer in the strategy and leadership sector has been instrumental in the successful establishment of knowledge management in sports organizations and by emphasize on localization in Iran. Baker [27] described one of the main pillars of human resource development as knowledge management training and job empowerment. Kirkman \& Rosen [29] suggested that empowerment can be created in the light of the principles of management and its consequences for knowledge management acquired. Razaghi et al. [30] stated that the factors studied, such as sharing knowledge culture and understanding of human resources of knowledge, training staff, appropriate incentives and stimulating the use of full capacity of people in human resources and communication channels, with the aim of transfer Knowledge is one of the factors that sports organizations can exploit from these findings to successfully deploy knowledge management in the organization. Also, Saharei [31], Rastadmehr et al. [8], Esfandiari \& Adabi [5], Mostafapour et al. (2012), Hasani \& Sheikh Esmaeili [2], Shaki et al. [22] state that the effect of knowledge management on empowerment employees is a positive and significant impact [32]. According to the results, it can be stated that knowledge management would be used as a measure to predict the empowerment of employees of the General Department of Sports and Youth in Mazandaran province.

\section{References}

1. Mohammadi AY (2017) Relationship between empowerment by learning and organizational commitment of personnel of Gilan department of sport and youth. International Journal of Sport Studies 7(3): 194-198.

2. Azarpira G, Yaghobi A, Forghani Ozrudi MB, Soleimani G (2013) The relationship between role clarity and job compatibility in the sport and physical education office. International Journal of Sport Studies 3(2): 194-198.

3. Forghani Ozrudi MB, Hasibi S, Shojaei V, Hami M (2016) Effect of employee empo wwerment on knowledge management in the departments of Sport and Youth Mazandaran. The First international conference of sport management. Shiraz, Iran.

4. Yaghobi A, Forghani Ozrudi MB (2015) Relationship between Organizational Culture and Knowledge Management with Professional Ethic in Physical Education Administrative Staff.

5. Esfandiari M, Adabi H (2016) Investigating the Relation between knowledge management practices and the psychological empowerment of human resources in municipality of Tehran. Urban Management Studies 8(25): 12-24.

6. Mogharah M, Madarshahian F, Aliabadi N, Rezayi N, Mohammadi A (2005) Job satisfaction of nurses in the hospital of Birjand. Journal of Birjand university of Medical Sciences 12(24): 24-37.

7. Adamson I (2005) Knowledge management: The next generation of TQM? Total Quality Management 16(8-9): 87-1000. 
8. Rastadmehr B, Ashraf Ganjouei F, Sajjadi Hazaveh S (2014) The effects empowering on knowledge management in sport federations staff. Sport Management Studies 6(25): 185-206.

9. Morrow JE (2012) Knowledge management operations. Headquarters, Department of the Army, Washington, USA.

10. Fazeli Deinan F, Safajani AM, Hosseini SE, Amirnejad S (2016) Investigating the relationship between effective factors on the successful establishment of knowledge management in selected sport organizations in Iran. Research on Sport Management and Motor Behavior 12(24): 3952.

11. Werner M (2002) The great paradox: Responsibility without empowerment, business horizons 35(5): 55-58.

12. Sheikhi PM (2015) Conflict and organizational empowerment. Nazar Arya, Tehran, Iran.

13. Bhatt G (2000) Organizing Knowledge in the Knowledge development cycle. Journal of Knowledge Management: Journal of Business Transformation 4(1): 15-26.

14. Naghibi H, Aghaei A (2008) The role of knowledge management in virtual organizations, research project with the supervision of the faculty of industry, Khajeh Nasir Al-Din Toosi University of Technology, Iran.

15. Bazgosha GR (2000) Personnel ability. Journal of the Book of Science and Technology.

16. Adli F (2005) Knowledge management, Moving to knowledge, Tehran, Andhis Metacognitive Publishing.

17. Akbarpour SM, Souri H, Mahdavi I (2006) Investigating the interaction between the process improvement process and the core knowledge management model, the fourth international management conference. Tehran, Iran.

18. Shafi A (2006) The relationship between employee empowerment and organizational learning at the national company for distribution of petroleum products, Master's thesis, Bushehr Azad University, Iran.

19. Kamkari K, Karimnejad N (2011) Validation and validation of staff empowerment questionnaire in physical education organization Master's thesis of physical education management, Islamic Azad University, Central Tehran Branch, Iran.

20. Farahani A, Falahati M (2007) Investigating the relationship between psychological factors of empowerment and organizational entrepreneurship among experts of physical education at university of Tehran. Research in Sport Sciences 5(15): 79-67.
21. Hasani K, Sheikhesmaeili S (2016) Knowledge management and employee empowerment. Kybernetes 45(2): 337-355.

22. Shakki MA, Garkaz M, Dahghan M (2016) The relationship between empowerment and knowledge management processes in esfahan oil refining company. International Research Journal of Management Sciences 4(6): 362-366.

23. Nourian I (2011) Investigating and analyzing the factors affecting the empowerment of employees of the Physical Education Organization of Ardebil province and introducing its improvement methods, National Conference on Physical Education and Sport Sciences, Islamic Azad University, Ayatollah Amoli Branch, Amol, Iran.

24. Ghorbanzadeh V, Khaleghinia S (2009) Effects of implicit knowledge transfer on employees' empowerment. Journal of Research in Human Resources Management 1(2): 85-105.

25. Keshavarz AH (2007) Barriers and facilitators of knowledge sharing in organizations, First National Conference on Knowledge Management, Razi International Con Center, Iran.

26. Saedi M, Nadalipour H (2008) Modeling the implementation of knowledge management in Iran, based on resource-based learning and source-oriented approach to participation in the IT field, research project under the supervision of Iran Khodro Co.

27. Becker BE (2008) High performance work system and firm Performance: A synthesis of research and managerial implications. Research in personnel and Human Resources Journal 16: 53-101.

28. Mousavi S (2012) Successful establishment of knowledge management in sport organizations with an emphasis on localization of Iran, International Conference on Sport Sciences, University of North.

29. Kirkman B, Rosen B (2009) Beyond self-management: The antecedent and consequences of team empowerment. Academy of management Journal 42(1): 58-71.

30. Razaghi ME, Moosavi SJ, Safania AM (2013) Successful knowledge management establishment in sport organizations with an emphasis on Iranian localization. International Journal of Sport Studies 3(1): 30-37.

31. Sahraei M (2013) The effect of empowerment on knowledge management in employees of physical education departments of Zanjan province. Master's Thesis, Islamic Azad University, Kurdistan Branch, Iraq.

32. Kamkari K, Shokrzadeh S (2010) Validation and validation of the personnel knowledge management questionnaire at Parsian bank (research project).
Creative Commons Attribution 4.0 International License

For possible submissions Click Here

\section{Submit Article}

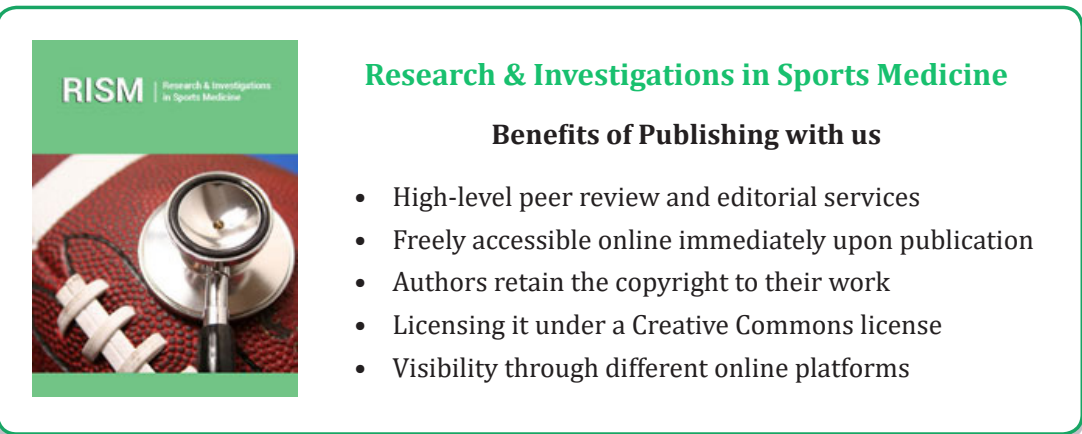

